\title{
Variations mensuelles sur trois années du spermogramme de béliers de race Djallonké en région forestière de la Côte d'Ivoire
}

\author{
Grah Nazaire Lavry ${ }^{1 *}$ M'Bétiégué Coulibaly ${ }^{2}$ \\ Atté Michel Offoumou ${ }^{3}$ Jacques Yao Datté ${ }^{3}$
}

\section{Mots-clés}

Ovin, bélier, Djallonké, récolte de sperme, fécondation, Côte d'Ivoire

Submitted: 7 June 2016

Accepted: 12 December 2016

Published: 30 January 2017

\section{Résumé}

Les variations mensuelles du volume, de la motilité massale et de la concentration de la semence ont été étudiées pendant trois années chez quarante béliers Djallonké de la zone forestière de Côte d'Ivoire. Deux éjaculats ont été récoltés par semaine à l'aide d'un vagin artificiel. Pour chaque année, le volume a été plus faible avant mai (de 0,55 $\pm 0,03$ à $0,75 \pm 0,04 \mathrm{ml}$ ) qu'après (de 0,61 $\pm 0,8$ à $0,9 \pm 0,61 \mathrm{ml}$ ) où les variations ont été significatives, sauf en 1993. La motilité massale n'a enregistré que des baisses significatives dans l'année (de 4,42 $\pm 0,06$ à 3,5 $\pm 0,2)$, mais avec un accroissement des valeurs entre avril et juillet (entre 3,85 $\pm 0,01$ et 4,45 $\pm 0,03$ ). La concentration a augmenté d'avril à août et significativement en juin et juillet [de 3,36 $\pm 0,12 \times 10^{9}$ à $3,8 \pm 0,12 \times 10^{9}$ spermatozoïdes $(\mathrm{spz}) / \mathrm{ml})]$. Les moyennes globales des trois années ont montré a) une augmentation du volume à partir de juin (de 0,71 $\pm 0,02$ à 0,77 $\pm 0,02$ $\mathrm{ml}$ ), et b) une baisse de la motilité massale significative de février à avril (de $4,28 \pm 0,06$ à $4,01 \pm 0,1)$, en juillet $(3,9 \pm 0,1)$, en septembre $(4,1 \pm 0,07)$ et en décembre $(3,92 \pm 0,1)$. La concentration a augmenté en juin (de 2,95 $\pm 0,05$ $\times 10^{9}$ à $\left.3,52 \pm 0,07 \times 10^{9} \mathrm{spz} / \mathrm{ml}\right)$ et juillet $\left(3,18 \pm 0,06 \times 10^{9} \mathrm{spz} / \mathrm{ml}\right)$. La variation de ces paramètres chez le Djallonké a donc été annuellement différente, non caractéristique et non-circannienne. Toutefois, la période entre avril et août a été plus favorable à l'augmentation des paramètres. Malgré les baisses significatives de la motilité massale, les paramètres ont été de bonne qualité pour I'insémination artificielle toute l'année.

- Pour citer cet article : Lavry G.N., Coulibaly M'B., Offoumou A.M., Datte J.Y, 2016. Three-year monthly variations in the spermogram of Djallonke-breed rams in the Cote d'Ivoire forest region. Rev. Elev. Med. Vet. Pays Trop., 69 (3): 111-116
1. Laboratoire d'endocrinologie et de biologie de la reproduction, UFR Biosciences, Université Félix Houphouët Boigny, 22 BP 582, Abidjan 22, Côte d'Ivoire.

2. Centre national d'insémination artificielle du Laboratoire central vétérinaire de Bingerville, Laboratoire national d'appui au développement agricole, Bingerville, Côte d'Ivoire.

3. Laboratoire de nutrition et pharmacologie, UFR Biosciences, Université Félix Houphouët Boigny, Abidjan, Côte d'Ivoire.

* Auteur pour la correspondance

Tél. : +22522412239

Portable : +22505587197/09523261

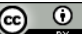

https://creativecommons.org/licenses/by/4.0/

\section{INTRODUCTION}

La production ovine en Côte d'Ivoire représentait 4,5 \% de la couverture en viande et abats en général en 2001 et $60 \%$ de la consommation nationale de viande de mouton (Leroy et al., 2002). Deux races ovines principales sont représentées dans le pays : la Sahélienne (Anonyme, 1995) et la Djallonké (Charray, 1986 ; Doutressoul, 1947 ; Vallerand et Branckaert, 1975). La Sahélienne est principalement constituée de moutons peuls originaires de la zone sahélienne du Niger et du Burkina Faso. Ce sont des moutons efflanqués et hauts sur pattes (taille de 65 à $75 \mathrm{~cm}$ ), pour un poids corporel à l'âge adulte de 30-50 kg. Le mouton Djallonké ou mouton Nain d'Afrique est de format plus petit : taille moyenne de $50 \mathrm{~cm}$, poids corporel de 20-30 kg pour la brebis et de 25-35 kg pour le bélier. Il représente la population de moutons la plus importante en Côte d'Ivoire (Anonyme, 1995). Par ailleurs, le Djallonké est rustique et tripanotolérant 
(Mawuena, 1987 ; Rombaut et Van Vlaenderen, 1976 ; Touré et Meyer, 1999), alors que le Peul est d'intégration plus récente. Les taux de fertilité et de prolificité du Djallonké sont respectivement de 86 à $100 \%$ et de 107 à $148 \%$ (Amegee, 1983 ; Rombaut et Van Vlaenderen, 1976).

Peu de travaux ont porté sur la reproduction des béliers de cette race, contrairement aux brebis (Asante et al., 1999 ; Awotwi et al., 2001 ; Berger, 1979). Certains travaux ont signalé la précocité de cette race (Adu et Olaloukou, 1979 ; Touré et Meyer, 1999) car elle produit ses premiers éjaculats à l'âge de 173,2 jours, pour un poids vif de 15,6 kg (Touré et Meyer, 1999). Les travaux de Chiboka (1980) sur la semence de Djallonké au Nigeria ont indiqué une amélioration significative de la motilité progressive en grande saison des pluies (d'avril à juin) par rapport à la grande saison sèche (de décembre à février), de 38,6 \% à 58,96\% ; ceci n'est pas le cas pour le volume et la concentration. Plus récemment Haye (2006), et Haye et al. (2004), en zones de savane arborée et forestière de la Côte d'Ivoire, ont trouvé chez des béliers Djallonké, respectivement âgés et jeunes, des volumes de 0,87 à $0,62 \mathrm{ml}$ et de 0,76 à $0,62 \mathrm{ml}$, une motilité massale de 2,67 à 2,8 et de 4 à 3,52 , et une concentration de $0,96 \times 10^{9}$ à $1,1 \times 10^{9} \mathrm{spz} / \mathrm{ml}$ et $2,51 \times 10^{9}$ à $2,94 \times 10^{9} \mathrm{spz} / \mathrm{ml}$. Selon ces résultats, le volume est meilleur chez les adultes dans les deux zones, au contraire de la concentration. Ces auteurs ont aussi rapporté que seule la motilité massale du sperme de béliers Djallonké âgés en zone forestière variait significativement dans l'année.

La présente étude, menée en zone forestière du sud de la Côte d'Ivoire chez le bélier Djallonké, visait une meilleure connaissance de l'évolution mensuelle du volume, de la motilité massale et de la concentration du sperme. Ces paramètres sont analysés en routine au laboratoire pour l'insémination artificielle. Une fonction a été recherchée à partir de valeurs standard pour déterminer la concentration quel que soit le taux de transmission. Ce travail a été intégré par la suite à des recherches sur l'effet de substances naturelles issues de la pharmacopée sur les paramètres de qualité du sperme de bélier (Djallonké et Ile-de-France), qui n'ont pu être finalisées qu'en 2014 (Lavry, 2014). L'objectif était d'améliorer la génétique et la productivité de la race Djallonké, après l'identification de géniteurs de référence et la constitution d'une banque de semences de qualité pour l'insémination artificielle. Par ailleurs, une régulation annuelle et des périodes favorables ou défavorables au spermogramme de béliers Djallonké ont été recherchées.

\section{- MATERIEL ET METHODES}

L'expérience a été réalisée pendant trois années $(1992,1993,1994)$ au Centre national d'insémination artificielle (CNIA) de Bingerville, à 15 kilomètres d'Abidjan, avec quarante béliers adultes de race Djallonké (Rombaut et Van Vlaenderen, 1976 ; Vallerand et Branckaert, 1975), indifféremment âgés de 1 à 5 ans et avec un poids vif de 35 à $49 \mathrm{~kg}$. Au cours de l'expérimentation, les animaux ont été vaccinés contre l'épididymite contagieuse ovine et la pasteurellose. Leur alimentation était à base de pâturage de Panicum maximum (poacée) avec une complémentation de son de riz, de tiges de mil et de sorgho, de fanes de niébé, et d'oligo-éléments sous forme de pierre à lécher.

Un éjaculat de sperme a été collecté deux fois par semaine au vagin artificiel, à l'aide d'une brebis boute-en-train en chaleurs ou non. Le volume du sperme a été relevé à travers le tube collecteur fixé à l'extrémité du vagin artificiel. La motilité massale, notée de 0 à 5 (Baril et al., 1993 ; Derivaux et Ectors, 1986 ; Evans et Maxwell, 1987), a été obtenue après observations à l'objectif 10 d'un microscope photonique (Will-Wetzlar) muni d'une plaque chauffante. La concentration a été déterminée par la méthode néphélométrique avec un spectrocolorimètre photoélectrique (Erma, model AE-1 $1 \mathrm{~N}$ ) à la longueur d'onde de $530 \mathrm{~nm}$. Le taux de transmission de lumière a été mesuré à travers une cuve contenant du sperme dilué à $4 \%$ dans une solution physiologique $(\mathrm{NaCl}, 1 \mathrm{~g} / 1000)$ formolée. Cette transmission correspondait à une concentration standard préalablement déterminée par numération directe à l'hématimètre (cellule de Mallasez), puis simultanément par le spectrocolorimètre qui a donné la transmission correspondante. La concentration a été ensuite calculée avec une fonction standard, tracée avec le logiciel Criket Graph de Macintosh (Lavry, 2014)

Les résultats ont été exprimés sous forme de moyenne mensuelle et d'écart-type. Les moyennes des mois ont été comparées avec le logiciel Stata version 9. Le degré de signification de différence (p) a été déterminé pour chaque paramètre, au seuil de $5 \%$. Les courbes de variation des paramètres ont été tracées avec le logiciel Excel avec des barres d'erreur à $5 \%$.

\section{RESULTATS}

\section{Volume}

Par année, la variation du volume, bien que mensuellement différente, a été dans l'ensemble plus faible avant mai qu'après (tableau I). La moyenne globale des trois années a baissé jusqu'en avril (de $0,71 \pm 0,02$ à $0,67 \pm 0,03 \mathrm{ml}$ ), puis a augmenté de juin à décembre (de $0,73 \pm 0,02$ à $0,81 \pm 0,02 \mathrm{ml})$. Cette variation globale a été significative $(\mathrm{p}<0,05)$ à la hausse, de juin à août, puis d'octobre à décembre. Les valeurs extrêmes ont été observées en octobre $(0,81 \pm 0,02 \mathrm{ml})$ et février $(0,65 \pm 0,2 \mathrm{ml})$. Les variations mensuelles ont montré dans l'ensemble, par année, une légère baisse entre février et mai, et une augmentation entre août et novembre, alors que les valeurs globales ont augmenté après le mois d'avril.

\section{Motilité massale}

Des fluctuations de la motilité massale ont été observées chaque année mais les variations n'ont pas été homogènes (tableau I). Ces variations étaient statistiquement significatives, notamment de 4,42 $\pm 0,06$ en janvier à 3,5 $\pm 0,2$ en septembre 1994. Les valeurs extrêmes ont été obtenues pendant des mois différents selon l'année. La motilité massale mensuelle moyenne de 4,28 $\pm 0,06$ en janvier a diminué, sauf en octobre $(4,3 \pm 0,04)$. Les moyennes des autres mois étaient statistiquement différentes $(\mathrm{p}<0,05)$ de février $(4,01 \pm 0,08)$ à avril $(4,01 \pm 0,1)$, puis en juillet $(3,9 \pm 0,1)$, septembre $(4,1 \pm 0,07)$ et décembre $(3,92 \pm 0,1)$. Les valeurs mensuelles extrêmes ont été observées en octobre $(4,3 \pm 0,04)$ et juillet $(3,9 \pm 0,1)$. Les variations de la motilité massale ont toutes montré une baisse en juillet et un accroissement général en octobre. L'accroissement maximal des moyennes en mai, juin et octobre s'est limité au niveau de la valeur initiale.

\section{Concentration}

\section{Données standard de détermination de la concentration}

Les valeurs standard de concentration déterminées simultanément à l'hématimètre et par néphélométrie ont été présentées dans le tableau II. Elles constituent, pour des raisons pratiques, la moyenne sur 32 échantillons. La courbe de la figure 1 a permis d'obtenir la fonction standard pour le calcul de la concentration : $\mathrm{Y}=1,2035 \times 10^{-3} \mathrm{X}^{2}-0,23 \mathrm{X}+12,41$. Cette fonction a donné par bélier les valeurs extrêmes de $1,7 \times 10^{9} \mathrm{spz} / \mathrm{ml}$ en mars 1994 et $7,9 \times 10^{9} \mathrm{spz} / \mathrm{ml}$ en mai 1992 , de même que plusieurs autres valeurs ne figurant pas dans le tableau II.

\section{Variations mensuelles de la concentration}

Par année, la concentration a varié différemment au cours de la période d'étude (tableau I). Toutefois, elle a augmenté dans l'ensemble 


\section{Tableau I}

Moyennes mensuelles au cours de trois années du volume, de la motilité massale et de la concentration en spermatozoïdes chez quarante béliers Djallonké au CNIA de Bingerville en Côte d'Ivoire

\begin{tabular}{|c|c|c|c|c|}
\hline Année & Mois & $\begin{array}{l}\text { Volume } \\
\text { (ml) }\end{array}$ & Motilité massale & $\begin{array}{l}\text { Concentration } \\
\left(\times 10^{9} \mathrm{spz} / \mathrm{ml}\right)\end{array}$ \\
\hline \multirow[t]{12}{*}{1992} & Janvier & $0,65 \pm 0,04^{a}$ & $4,32 \pm 0,06^{a}$ & $3,5 \pm 0,08^{a}$ \\
\hline & Février & $0,63 \pm 0,03^{a}$ & $3,9 \pm 0,15^{b}$ & $3,7 \pm 0,14 \mathrm{a}^{*}$ \\
\hline & Mars & $0,62 \pm 0,04^{a}$ & $3,4 \pm 0,2^{b^{*}}$ & $3,46 \pm 0,16^{a}$ \\
\hline & Avril & $0,55 \pm 0,03 b^{*}$ & $3,85 \pm 0,1^{b}$ & $3,62 \pm 0,09^{a}$ \\
\hline & Mai & $0,84 \pm 0,06^{b}$ & $4,35 \pm 0,05^{a}$ & $3,68 \pm 0,24^{a}$ \\
\hline & Juin & $0,75 \pm 0,03{ }^{a}$ & $4,45 \pm 0,03 a^{a^{*}}$ & $3,64 \pm 0,12^{a}$ \\
\hline & Juillet & $0,88 \pm 0,09 b^{*}$ & $4,05 \pm 0,2^{\mathrm{a}}$ & $3,36 \pm 0,12^{a}$ \\
\hline & Août & $0,87 \pm 0,03 b$ & $4,15 \pm 0,1^{a}$ & $3,37 \pm 0,15^{a}$ \\
\hline & Septembre & $0,73 \pm 0,03 a^{a}$ & $4,07 \pm 0,1^{b}$ & $2,7 \pm 0,1 b^{*}$ \\
\hline & Octobre & $0,84 \pm 0,03 b$ & $4,4 \pm 0,1^{a}$ & $2,74 \pm 0,14^{b}$ \\
\hline & Novembre & $0,8 \pm 0,05^{b}$ & $4,35 \pm 0,07^{a}$ & $3,8 \pm 0,22^{a}$ \\
\hline & Décembre & $0,8 \pm 0,02^{b}$ & $4 \pm 0,2^{a}$ & $3,3 \pm 0,15^{a}$ \\
\hline \multirow[t]{12}{*}{1993} & Janvier & $0,79 \pm 0,03^{a}$ & $4,17 \pm 0,2^{a}$ & $2,89 \pm 0,06^{a}$ \\
\hline & Février & $0,65 \pm 0,03 b^{*}$ & $3,9 \pm 0,2^{a}$ & $2,87 \pm 0,07^{a}$ \\
\hline & Mars & $0,7 \pm 0,02 b$ & $4,21 \pm 0,07^{a}$ & $2,96 \pm 0,09 a$ \\
\hline & Avril & $0,75 \pm 0,04^{a}$ & $3,9 \pm 0,15^{a}$ & $2,69 \pm 0,06^{b}$ \\
\hline & Mai & $0,66 \pm 0,03 b$ & $4,33 \pm 0,06^{a}$ & $2,61 \pm 0,08 b^{b^{*}}$ \\
\hline & Juin & $0,77 \pm 0,04^{a}$ & $4,3 \pm 0,06^{a}$ & $3,8 \pm 0,12 b^{*}$ \\
\hline & Juillet & $0,83 \pm 0,04^{a^{*}}$ & $3,76 \pm 0,14 \mathrm{~b}^{*}$ & $3,32 \pm 0,1 b$ \\
\hline & Août & $0,75 \pm 0,03^{a}$ & $4,05 \pm 0,14^{a}$ & $3,03 \pm 0,12^{a}$ \\
\hline & Septembre & $0,73 \pm 0,25^{a}$ & $4,4 \pm 0,05^{a}$ & $3,66 \pm 0,13^{b}$ \\
\hline & Octobre & $0,75 \pm 0,02 \mathrm{a}$ & $4,42 \pm 0,03 \mathrm{a}^{*}$ & $3,01 \pm 0,07^{a}$ \\
\hline & Novembre & $0,77 \pm 0,03^{a}$ & $4,3 \pm 0,07^{a}$ & $3,11 \pm 0,1^{\mathrm{a}}$ \\
\hline & Décembre & $0,76 \pm 0,02^{a}$ & $3,95 \pm 0,12^{a}$ & $2,8 \pm 0,1^{a}$ \\
\hline \multirow[t]{12}{*}{1994} & Janvier & $0,64 \pm 0,03^{a}$ & $4,42 \pm 0,06^{a^{*}}$ & $2,51 \pm 0,05^{a}$ \\
\hline & Février & $0,69 \pm 0,05^{a}$ & $4,27 \pm 0,1^{\mathrm{a}}$ & $2,98 \pm 0,12^{a}$ \\
\hline & Mars & $0,64 \pm 0,05^{a}$ & $4,35 \pm 0,07^{a}$ & $2,66 \pm 0,2^{a}$ \\
\hline & Avril & $0,65 \pm 0,05^{a}$ & $4,37 \pm 0,07^{a}$ & $2,74 \pm 0,13^{a}$ \\
\hline & Mai & $0,75 \pm 0,05^{b}$ & $4 \pm 0,14^{b}$ & $3,64 \pm 0,12 b^{*}$ \\
\hline & Juin & $0,79 \pm 0,05 b$ & $4,05 \pm 1,1^{b}$ & $2,88 \pm 0,1 \mathrm{~b}$ \\
\hline & Juillet & $0,66 \pm 0,05^{a}$ & $3,85 \pm 0,1 b$ & $2,74 \pm 0,05 b$ \\
\hline & Août & $0,61 \pm 0,8^{a^{*}}$ & $4,22 \pm 0,1^{\mathrm{a}}$ & $2,55 \pm 0,04^{a}$ \\
\hline & Septembre & $0,79 \pm 0,07^{b}$ & $3,5 \pm 0,2 b^{*}$ & $2,21 \pm 0,05 b^{*}$ \\
\hline & Octobre & $0,9 \pm 0,61^{b}$ & $4,1 \pm 0,14^{b}$ & $2,46 \pm 0,07^{a}$ \\
\hline & Novembre & $0,85 \pm 0,06^{b^{*}}$ & $3,77 \pm 0,12^{b}$ & $2,53 \pm 0,06^{a}$ \\
\hline & Décembre & $0,79 \pm 0,06^{b}$ & $3,77 \pm 0,14^{b}$ & $3,1 \pm 0,14^{a}$ \\
\hline \multirow{12}{*}{$\begin{array}{l}\text { Moyennes globales } \\
(1992,1993 \text { et 1994) }\end{array}$} & Janvier & $0,71 \pm 0,02^{a}$ & $4,28 \pm 0,06^{a}$ & $2,95 \pm 0,05^{a}$ \\
\hline & Février & $0,65 \pm 0,2^{a^{*}}$ & $4,01 \pm 0,08^{b}$ & $3,11 \pm 0,07^{a}$ \\
\hline & Mars & $0,66 \pm 0,02^{a}$ & $4,03 \pm 0,07^{b}$ & $3,01 \pm 0,08^{a}$ \\
\hline & Avril & $0,67 \pm 0,03^{a}$ & $4,01 \pm 0,1^{b}$ & $2,95 \pm 0,07^{a}$ \\
\hline & Mai & $0,73 \pm 0,02^{a}$ & $4,25 \pm 0,06^{a}$ & $3,16 \pm 0,1^{a}$ \\
\hline & Juin & $0,77 \pm 0,02 b$ & $4,27 \pm 0,04^{a}$ & $3,52 \pm 0,07 b^{*}$ \\
\hline & Juillet & $0,8 \pm 0,03 b$ & $3,9 \pm 0,1 \mathrm{~b}^{*}$ & $3,18 \pm 0,06^{b}$ \\
\hline & Août & $0,77 \pm 0,02^{b}$ & $4,12 \pm 0,1^{a}$ & $2,99 \pm 0,07^{a}$ \\
\hline & Septembre & $0,75 \pm 0,03$ a & $4,1 \pm 0,07^{b}$ & $3,03 \pm 0,1^{a}$ \\
\hline & Octobre & $0,81 \pm 0,02 b^{*}$ & $4,3 \pm 0,04 a^{*}$ & $2,8 \pm 0,05 \mathrm{a}^{*}$ \\
\hline & Novembre & $0,8 \pm 0,02 b$ & $4,16 \pm 0,06^{a}$ & $3,13 \pm 0,1^{a}$ \\
\hline & Décembre & $0,77 \pm 0,02^{b}$ & $3,92 \pm 0,1^{b}$ & $3,02 \pm 0,1^{a}$ \\
\hline
\end{tabular}

CNIA : Centre national d'insémination artificielle ; spz : spermatozoïdes

Les valeurs suivies de ${ }^{\text {a }}$ ne sont pas significatives par rapport au mois de janvier, au seuil de $5 \%$.

* Valeurs extrêmes 


\section{Tableau II}

Valeurs standard de transmission et de concentration du sperme de bélier Djallonké en Côte d'Ivoire à la longueur d'onde de $530 \mathrm{~nm}$ (photomètre ERMA)

\begin{tabular}{|c|c|c|c|c|c|c|c|c|c|c|c|c|c|c|c|}
\hline \multicolumn{16}{|l|}{ Transmission (\%) } \\
\hline 73 & 76 & 66 & 62 & 59 & 57 & 54 & 52 & 50 & 48 & 46 & 44 & 42 & 40 & 38 & 36 \\
\hline \multicolumn{16}{|c|}{ Concentration $\left(\times 10^{9} \mathrm{spz} / \mathrm{ml}\right)$} \\
\hline 1,5 & 2 & 2,25 & 2,5 & 2,75 & 3 & 3,25 & 3,5 & 3,75 & 4 & 4,25 & 4,5 & 4,75 & 5 & 5,25 & 5,5 \\
\hline
\end{tabular}

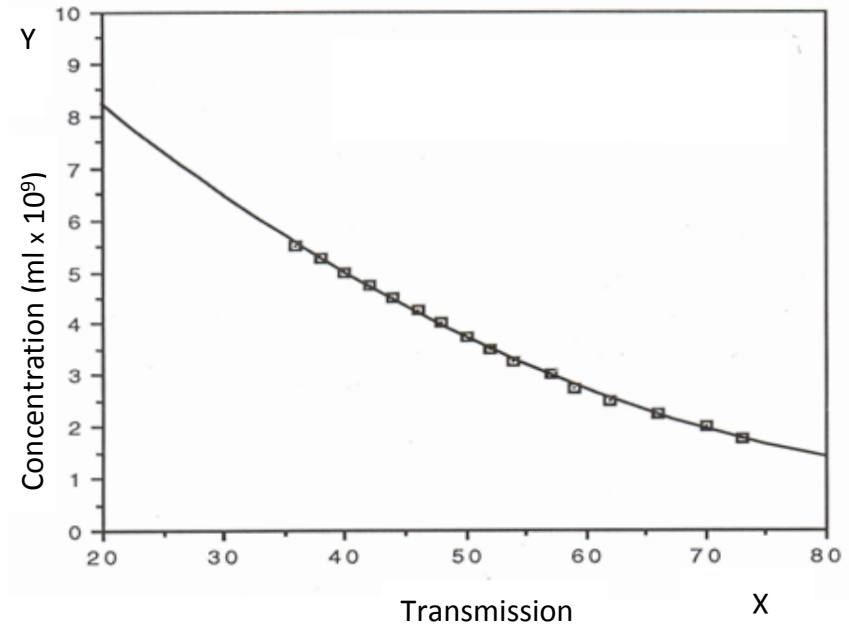

Figure 1 : courbe standard de la concentration (y) du sperme en fonction de la transmission $(x)$ de la lumière au spectrophotomètre, réalisée avec le logiciel Cricket graph de Macintosh qui a donné la fonction, $Y=1,2035 \times 10^{-3} X^{2}-0,23 X+12,41$ avec un coefficient de corrélation $R^{2}=0,999$.

entre mai et juillet, et significativement de $2,89 \pm 0,06 \times 10^{9}$ à $3,8 \pm 0,12 \times 10^{9} \mathrm{spz} / \mathrm{ml}$ en juin, et à $3,32 \pm 0,1 \times 10^{9} \mathrm{spz} / \mathrm{ml}$ en juillet 1993, ainsi que pour ces mêmes mois en 1994. Des baisses n'ont été significatives qu'en 1992. La concentration mensuelle globale (tableau I) de $2,95 \pm 0,05 \times 10^{9} \mathrm{spz} / \mathrm{ml}$ en janvier est passée à $3,16 \pm 0,1 \times 10^{9} \mathrm{spz} / \mathrm{ml}$ en mai et à $3,18 \pm 0,06 \times 10^{9} \mathrm{spz} / \mathrm{ml}$ en juillet. Cette variation a significativement augmenté $(p<0,05)$ en juin et juillet. Des variations extrêmes ont été observées en juin $\left(3,52 \pm 0,07 \times 10^{9} \mathrm{spz} / \mathrm{ml}\right)$ et octobre $\left(2,8 \pm 0,05 \times 10^{9} \mathrm{spz} / \mathrm{ml}\right)$.

Les variations mensuelles du volume, de la motilité massale et de la concentration du sperme de bélier Djallonké ont différé pendant les trois années aussi bien au cours de chacune d'elles que pour les valeurs globales. Pour ces dernières, le volume et la concentration ont principalement augmenté dans l'année, alors que la motilité massale a diminué. Par ailleurs, dans la période entre avril et août une croissance des paramètres a été observée.

\section{- DISCUSSION}

Les variations mensuelles pendant trois années du volume, de la motilité massale et de la concentration, évaluées pour l'insémination artificielle du sperme de bélier Djallonké en zone forestière de la Côte d'Ivoire, ont été différentes d'une année à l'autre. Ceci traduit une grande variabilité, comme l'ont rapporté différents auteurs (Derivaux et Ectors, 1986 ; Baril et al., 1993 ; Dadoune et Demoulin, 2001). Ces variations mensuelles ont montré que ces paramètres n'ont pas subi de régulation cyclique au cours des trois années. Cette observation a été faite chez plusieurs races ovines des climats tropicaux (Chemineau et al., 2001 ; Pedigo et al., 1989). Au regard de ces paramètres, il n'y a pas eu de régulation biologique circannienne du sperme de Djallonké. Ces paramètres ont tous varié significativement $(\mathrm{p}<0,05)$ au moins deux fois chaque année (à la hausse ou à la baisse), probablement en raison des différentes variations de facteurs environnementaux (par exemple climat, température, photopériode, végétation) au cours de chaque année. Cependant, malgré ces différences annuelles, une croissance générale des paramètres a été observée entre avril et juillet, puis en octobre et novembre. Ceci pourrait suggérer une certaine variation périodique de ces paramètres si des moyennes par groupe de mois étaient réalisées, notamment par saison (Baril et al., 1993 ; Dadoune et Demoulin, 2001).

L'analyse de la variation des moyennes globales des trois années a montré que la motilité massale a baissé, dénotant une évolution défavorable après le mois de janvier. Le volume et la concentration ont varié significativement à la hausse et la période de mai à juillet a été la plus favorable. Ces variations sont différentes des résultats de Haye (2006) qui, chez cette race et dans la même localité, a indiqué des variations significatives à la hausse et à la baisse de tous ces paramètres. Il a aussi, à la différence du présent travail, rapporté des valeurs extrêmes de $0,77 \mathrm{ml}$ en janvier et $0,60 \mathrm{ml}$ en juillet pour le volume, 3,96 en août et 3,50 en septembre pour la motilité massale, et $2,36 \times 10^{9} \mathrm{spz} / \mathrm{ml}$ en mai et $3,46 \times 10^{9} \mathrm{spz} / \mathrm{ml}$ en septembre pour la concentration. Ces moyennes globales des paramètres ont varié différemment dans l'année, mais un accroissement des valeurs a été observé de mai à juillet et secondairement entre octobre et novembre. Au regard de ces variations mensuelles globales et par année, ces périodes de mai à juillet, puis d'octobre et de novembre sont apparues comme favorables au spermogramme du bélier Djallonké. La hausse des paramètres en juillet est conforme aux résultats de Haye chez le Djallonké (Haye, 2006), et de Issa et al. (2001) chez des béliers Peuls bicolores au Niger. Ces variations mensuelles ont traduit une influence différente des facteurs environnementaux sur la qualité du sperme du Djallonké, comme l'ont signalé Baril et al. (1993), Lodge et Salibury (1970), et Pellicer-Rubio et Combarnous (1998). Mais, une augmentation caractéristique s'est produite en juillet.

La bonne qualité des paramètres du sperme étudiés est déterminée par un volume moyen de $0,8 \mathrm{ml}$ (extrêmes de 0,5 à $2 \mathrm{ml}$ ), une motilité massale égale ou supérieure à 3,5 , une concentration comprise entre $2 \times 10^{9}$ et $6 \times 10^{9} \mathrm{spz} / \mathrm{ml}$ (Colas, 1980 ; Evans et Maxwell, 1987 ; Pedigo et al., 1989). Les présents résultats ont montré que, malgré les modifications à la baisse de la motilité massale, les paramètres étudiés n'affectaient pas dans l'année la bonne qualité du sperme pour l'insémination artificielle. Cette bonne qualité a déjà été signalée chez le Djallonké (Haye et al., 2004 ; Haye, 2006) et chez le Peul bicolore (Issa et al., 2001). En conséquence, dans la zone forestière de la Côte d'Ivoire la qualité du sperme de bélier Djallonké pour l'insémination artificielle n'a pas été dégradée au cours de l'année. En effet, le volume, la motilité massale et la concentration ont donné des valeurs bonnes, toute l'année, pour l'insémination artificielle. 
$\mathrm{Au}$ regard des résultats obtenus, les variations des moyennes mensuelles constatées des trois années seraient donc dues soit à l'état de santé ou de nutrition des béliers (Craplet et Thibier, 1980 ; Derivaux et Ectors, 1986), soit à des phénomènes environnementaux (Baril et al., 1993 ; Chemineau et al., 2001 ; Lodge et Salibury, 1970). Dans la mesure où la photopériode ne varie pratiquement pas toute l'année en zone tropicale guinéenne, la variation des facteurs saisonniers et climatiques, avec probablement leurs conséquences sanitaires et nutritionnelles (Craplet et Thibier, 1980), seraient à la base des modifications mensuelles enregistrées au cours de l'année au niveau des paramètres étudiés chez le bélier Djallonké. En effet, il est admis que les facteurs climatiques influencent directement la végétation et la disponibilité de la nourriture, de même que l'abri pour les animaux. Cela influence indirectement la reproduction (Lodge et Salibury, 1970). Dans la présente étude, l'influence de l'alimentation a dû provenir de la disponibilité de Panicum maximum qui constituait le pâturage.

\section{CONCLUSION}

Après trois années d'étude des paramètres du sperme (volume, motilité massale, concentration) de béliers Djallonké récolté par vagin artificiel, une variation mensuelle différente de tous ces paramètres a été observée d'une année à l'autre. L'évolution de ces paramètres a montré qu'ils ne sont pas soumis à une variation mensuelle caractéristique dans l'année chez cette race. Les moyennes globales des trois années ont montré que la motilité massale a baissé après janvier, alors que le volume et la concentration ont augmenté de juin à juillet. Toutefois, que ce soit par année ou avec les valeurs globales, la qualité du volume, de la motilité massale et de la concentration est restée bonne toute l'année pour l'insémination artificielle chez le bélier Djallonké. Cependant, la comparaison des courbes de variations mensuelles des valeurs globales des trois paramètres a permis d'identifier une période favorable au spermogramme située d'avril à juillet.

\section{REFERENCES}

Adu I.F., Olaloukou A., 1979. A note on nutrition during late pregnancy in West African Dwarf Sheep. Res. Vet. Sci., 4 (1): 104-106

Amegee Y., 1983. Study on the prolificacy of Djallonke sheep in a village environment in Togo. Rev. Elev. Med. Vet. Pays Trop., 36 (1): 85-90

Anonyme, 1995. Rapport annuel. Ministère de la Production animale, Abidjan, Côte d'Ivoire, 36 p.

Asante Y.A., Oppong-Anane K., Awotwi E.K., 1999. Djallonke and Sahelian ewes and their lamb during the first 24 hours post-partum. Appl. Anim. Behav. Sci., 65: 53-61

Awotwi E.K., Canacoo E.A., Adogla-Bassa T., Oppong-Anane K., Oddoye E.O.K., 2001. The effect of age at mating on the behavioural interaction between primiparous Djallonke ewes and their lambs at 36 hours post-partum. Anim. Behav. Sci., 75: 47-54

Baril G., Chemineau P., Cognie Y., Guerin Y., Leboeuf B., Orgueur P., Vallet J.-C., 1993. Manuel de formation pour l'insémination artificielle chez les ovins et les caprins. FAO, Rome, Italie, 231 p. (Production et santé animales ; 83

Berger Y., 1979. Results of four years' study on the Jallonke sheep breed in Ivory Coast. Rev. Elev. Med. Vet. Pays Trop., 33 (1): 71-78

Charray J., 1986. The performance of West Africa dwarf ewes managed with two different accelerated lambing programs. Rev. Elev. Med. Vet. Pays Trop., 39 (1): 151-160
Chemineau P., Gognie Y., Thimonier J., 2001. La maîtrise de la reproduction des mammifères domestiques. In : Reproduction chez es mammifères et l'homme. INRA, Versailles, France, p. 792-815 (Ellipses)

Chiboka O., 1980. Semen characteristics of West African Dwarf ram. Anim. Reprod. Sci., 3: 247-252

Colas G., 1980. Variation saisonnière de la qualité du sperme chez le bélier Ile-de-France. I. Etude de la morphologie cellulaire et de la motilité massale. Reprod. Nutr. Develop., 20 : 1789-1799

Craplet C., Thibier M., 1980. Le mouton. Traité d'élevage moderne, tome IV, 4 e édn. Vigot Frères, Paris, France, 568 p.

Dadoune P., Demoulin A., 2001. Structure et fonction du testicule. In : La reproduction chez les Mammifères et l'Homme. INRA, Versailles, France, p. 256-315

Derivaux J., Ectors F., 1986. Reproduction chez les animaux domestiques, $3^{\mathrm{e}}$ édn. Cabay, Louvain-La-Neuve, Belgique, $1096 \mathrm{p}$.

Doutressoul E., 1947. Elevage en Afrique occidentale française. Larose, Paris, France, 228 p.

Evans G., Maxwell W.M.C., 1987. Salamon's artificial insemination of sheep and goats. Butterworths, London, UK, 194 p.

Haye A., 2006. Les paramètres quantitatifs et qualitatifs du sperme du bélier de race Djallonké en fonction des saisons. Thèse Doct., Université de Cocody, Abidjan, Côte d'Ivoire, 140 p.

Haye A., M’Bétiégué C., Nazaire L.N., Tanon B., 2004. Evaluation de la qualité du sperme du bélier de la race Djallonké en région de savane humide de Côte d'Ivoire. Agro. Afr., 16 : 37-47

Issa M., Yenikoyé A., Marichatou H., Banoin M., 2001. Spermogram of bicolor Peul and Touareg rams: genetic type and seasonal influence. Rev. Elev. Med. Vet. Pays Trop., 54 (3-4): 269-275

Lavry G.N., 2014. Etude des paramètres de qualité du sperme de béliers Djallonké en zone forestière et de béliers Ile-de-France : étude intégrée de l'effet de Costus afer (Zingiberaceae). Thèse Doct. Sciences naturelles, Université Félix Houphouët Boigny, Abidjan, Côte d'Ivoire, $164 \mathrm{p}$.

Leroy P., Koné K., Leroy E., 2002. Etude des systèmes d'amélioration génétique des bovins et ovins en Côte d'Ivoire. Rapport provisoire du projet Appui à I'amélioration génétique du cheptel. Ministère de la Production animale et des Ressources halieutiques, Abidjan, Côte d'Ivoire, $75 \mathrm{p}$.

Lodge J.R., Salibury G.W., 1970. Seasonal variation and male reproduction efficiency. In: The Testis, Vol. III (Eds. Johnson A.D. Gomez W.R., Vandermark N.L.). London, UK, Academic Press, p. 137167

Mawuena K., 1987. High level of tolerance to trypanosomiasis of West African Dwarf sheep and goats from South Guinean countries of Togo. Comparison with trypanotolerant cattle. Rev. Elev. Med. Vet. Pays Trop., 40 (1): 55-58

Pedigo G.N., Vernon W.M., Curry E.T., 1989. Characterization of a computerized semen analysis system. Fertil. Steril., 52 (4): 659-666

Pellicer-Rubio M.T., Combarnous Y., 1998. Deterioration of goat spermatozoa in skimmed milk-based extenders as a result of oleic acid released by the bulbourethral lipase BUSgp60. J. Reprod. Fert., 112: 95-105

Rombaut D., Van Vlaenderen G., 1976. Djallonke sheep of Ivory Coast in village environment. Behaviour and feeding. Rev. Elev. Med. Vet. Pays Trop., 29 (2): 157-172

Touré E., 1977. Trypanotolerance. Review on actual knowledge. Rev. Elev. Med. Vet. Pays. Trop., 30 (2): 157-174

Touré S.M., Meyer C., 1999. Evolution corporelle, testiculaire et comportementale chez l'agneau Djallonké. Agro. Afr., 2: 45-51

Vallerand F., Branckaert R., 1975. Djallonke sheep breed in Cameroon, zootechnical possibilities, breeding conditions, future. Rev. Elev. Med. Vet. Pays Trop., 28 (4): 523-545 


\section{Summary}

Lavry G.N., Coulibaly M'B., Offoumou A.M., Datté J.Y. Three-year monthly variations in the spermiogram of Djallonke-breed rams in the Cote d'Ivoire forest region

Monthly variations in the volume, mass motility and semen concentration were studied over three years in forty Djallonke rams in the forest area of Cote d'Ivoire. Two ejaculates were harvested weekly using an artificial vagina. For each year, the volume was lower before May (from $0.55 \pm 0.03$ to 0.75 $\pm 0.04 \mathrm{ml}$ ) than after (from $0.61 \pm 0.8$ to $0.9 \pm 0.61 \mathrm{ml}$ ), when changes were significant except in 1993. The mass motility showed only significant decreases in the year (from 4.42 \pm 0.06 to $3.5 \pm 0.2$ ), but with an increase between April and July (between $3.85 \pm 0.01$ and $4.45 \pm 0.03$ ). The concentration increased from April to August and significantly in June and July [from $3.36 \pm 0.12 \times 10^{9}$ to $3.8 \pm 0.12 \times 10^{9}$ spermatozoa $(\mathrm{spz}) / \mathrm{ml}$. Overall averages for the three years showed i) an increase in volume from June (from $0.71 \pm 0.02$ to 0.77 $\pm 0.02 \mathrm{ml})$, and ii) a significant decrease in mass motility from February to April (from $4.28 \pm 0.06$ to $4.01 \pm 0.1$ ), in July $(3.9 \pm 0.1)$, in September $(4.1 \pm 0.07)$ and in December $(3.92 \pm 0.1)$. The concentration increased in June (from 2.95 $\pm 0.05 \times 10^{9}$ to $\left.3.52 \pm 0.07 \times 10^{9} \mathrm{spz} / \mathrm{ml}\right)$ and in July $(3.18$ $\left.\pm 0.06 \times 10^{9} \mathrm{spz} / \mathrm{ml}\right)$. The variation of these parameters in Djallonke was thus annually different, non-characteristic and with no annual cycle. However, the period between April and August was more favorable to the increase of the parameters. Despite significant decreases in mass motility, the parameters were of good quality for artificial insemination throughout the year.

Keywords: sheep, ram, Djallonke, semen collection, fertilization, Cote d'Ivoire

\section{Resumen}

Lavry G.N., Coulibaly M'B., Offoumou A.M., Datté J.Y. Variaciones mensuales de tres años en parámetros del espermograma de semen de caneros de raza Djallonke en la región boscosa de Cote d'Ivoire

Se estudiaron variaciones mensuales en el volumen, la motilidad de masa y la concentración de semen, durante tres años, en cuarenta carneros Djallonke en la zona boscosa de Cote d'Ivoire. Se colectaron dos eyaculados semanalmente, usando una vagina artificial. Cada año, el volumen fue menor antes de mayo (de 0,55 $\pm 0,03$ a 0,75 $\pm 0,04 \mathrm{ml}$ ) que después (de $0,61 \pm 0,8$ a $0,9 \pm 0,61 \mathrm{ml})$, cuando los cambios fueron significativos, excepto en 1993. La motilidad de masa mostró únicamente disminuciones significativas en el año (de 4,42 $\pm 0,06$ a $3,5 \pm 0,2$ ), pero con un aumento entre abril y julio (de 3,85 $\pm 0,01$ a 4,45 \pm 0,03). La concentración aumentó de abril a agosto, y significativamente en junio y julio [de 3,36 $\pm 0,12 \times 10^{9}$ a 3,8 $\pm 0,12 \times 10^{9}$ espermatozoides $(\mathrm{spz}) / \mathrm{ml}$. Los promedios generales para los tres años son: a) un aumento en volumen a partir de junio (de 0,71 $\pm 0,02$ a $0,77 \pm 0,02 \mathrm{ml}), y$ b) un aumento significativo en motilidad de masa de febrero a abril (de 4,28 $\pm 0,06$ a 4,01 $\pm 0,1$ ), en julio $(3,9 \pm 0,1)$, en setiembre $(4,1 \pm 0,07)$ y en diciembre $(3,92 \pm 0,1)$. La concentración aumentó en junio (de 2,95 $\pm 0,05 \times 10^{9}$ a $\left.3,52 \pm 0,07 \times 10^{9} \mathrm{sptz} / \mathrm{ml}\right)$ y en julio $(3,18$ $\left.\pm 0,06 \times 10^{9} \mathrm{sptz} / \mathrm{ml}\right)$. La variación anual de estos parámetros en Djallonke fue por lo tanto diferente, no característica y sin un ciclo anual. Sin embargo, el periodo entre abril y agosto fue más favorable al aumento de los parámetros. A pesar de disminuciones significativas de la motilidad de masa, los parámetros fueron de buena calidad para la inseminación artificial a lo largo del año.

Palabras clave: ovino, morueco, Djallonke, recolección de semen, fecundación, Cote d'Ivoire 